\title{
Percutaneous Endoscopic Lumbar Discectomy for L5-S1 Foraminal Disc Herniation with Superior Migration using Contralateral Interlaminar Approach: A Technical Case Report
}

\author{
Keun Lee, Hyeun-Sung Kim², Jee-Soo Jang ${ }^{2}$, Yong-Hun Pee ${ }^{1}$, Jin-Uk Kim³, Jun-Ho Lee ${ }^{3}$, Il-Tae Jang ${ }^{4}$ \\ ${ }^{1}$ Department of Neurosurgery, Nanoori Jooan Hospital, Incheon, \\ 'Department of Neurosurgery, Nanoori Suwon Hospital, Suwon, \\ ${ }^{3}$ Department of Neurosurgery, Nanoori Incheon Hospital, Incheon, \\ ${ }^{4}$ Department of Neurosurgery, Nanoori Gangnam Hospital, Seoul, Korea
}

Corresponding Author: Hyeun-Sung Kim, MD, PhD

Department of Neurosurgery, Nanoori Suwon Hospital, 295, Jungbudaero, Yeongtong-gu, Suwon-si 16503, Korea

Tel: $+82-2-1688-9797$

Fax: +82-31-8065-9797

E-mail: neuros@hanmail.net

Received: April 25, 2016

Revised: May 16, 2016

Accepted: July 7, 2016

\begin{abstract}
In cases of L5-S1 foraminal disc herniation with superior migration, it is very difficult to access using rigid endoscope. We attempted a percutaneous endoscopic lumbar discectomy (PELD) via a contralateral interlaminar approach to remove the symptomatic disc and preserve the functional structures. Between January 2013 and January 2014, five patients who received PELD for the disc herniation via the approach were included in this study. Through the approach, we could expose the exiting nerve root without structural damage. We confirmed the appropriate decompression of the lesion with an immediate postoperative MRI, and the clinical outcome was evaluated using a visual analog scale (VAS) score. In all cases, the herniation was removed appropriately. The mean pre-operative VAS score was decreased at the post-operative state, from $7.8 \pm 0.84$ to $1.4 \pm 0.55$. We obtained excellent clinical outcomes in treating the migrated disc herniation at the level using PELD via a contralateral interlaminar approach.
\end{abstract}

Key Words: Contralateral, Lumbar disc herniation, Foraminal

\section{INTRODUCTION}

In a treatment of radiating pain related to the lumbar disc herniation, microscopic lumbar discectomy has been considered the gold standard"). However, due to the development of relevant equipment, such as a drill, forcep, laser, radiofrequency probe, and high-resolution optic endoscope, it has become possible to treat most lumbar disc herniations with percutaneous endoscopic lumbar discectomy (PELD) $)^{4,5,9,12,13)}$.

In cases of L5-S1 foraminal disc herniation with superior migration, both transforaminal and ipsilateral interlaminar approaches have technical difficulties. An appropriate endoscopic approach at the herniation has not been reported. In order to approach the contralateral foramen at the level, we attempted new approach that passed the interlaminar space from contralateral side to ipsilateral one of the lesion and present the approach.

\section{CASE REPORT}

Five patients complaining radiating pain were included between January 2013 and January 2014. The lesion of the patients was L5-S1 foraminal disc herniation with superior migration. The radiating pain of them was refractory to conservative treatment for more than six weeks. They underwent PELD via a contralateral interlaminar approach. The clinical outcomes were evaluated using visual analog scale (VAS) score and all patients were checked pre- and post-operative MRI to assess the decompression of the lesion.

\section{Operative techniques}

Prior to the operation, patients received preventive antibiotics and were prepared in a prone position on a radiolucent table. 
The operation was performed with conscious sedation using midazolam and fentanyl. In order to avoid injuries to exiting and traversing nerve roots during the approach, the operation was proceeded with continuous feedback from the patient. C-arm guidance was used to identify the L5-S1 disc space. The skin was marked at the midline of disc space. Discography was performed before insertion of working channel. 0.8\% indigo carmine (Carmine, Korea United Pharmaceutical, Yoenki, Korea) mixed with contrast (lobrix injection, Taejoon Pharm, Korea) were used for discography.

An 18-gauge spinal needle was inserted at the point which was about $2-3 \mathrm{~cm}$ away on the contralateral side of vertebral midline with an approximately 45-degree angle. After passing an interspinous ligament, the needle was docked at the medial border of inferior facet. A guide wire was inserted through the needle and then the needle was removed. An obturator was inserted just beyond the ligamentum flavum and then the wire was removed. A working channel was inserted through the obturator. An obturator was removed and then endoscope was inserted. The ligamentum flavum was splitted with a probe. Bevel of the working channel was penetrated through the splitted point of the ligamentum flavum and was rotated (Fig. 1). The splitted ligamentum flavum was pushed down, protecting a traversing nerve root and surrounding structures.

When the ligamentum flavum was splitted, a contralateral foraminal area was exposed. The epidural fat was dissected with a radiofrequency electrode (Ellman International, Hewlett, NY,

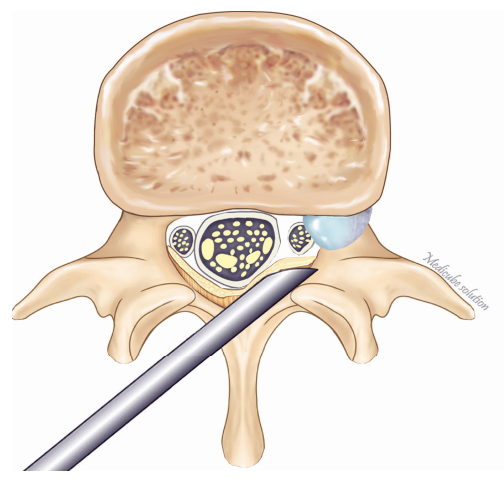

Fig. 1. Docking of working channel at the medial border of inferior facet.

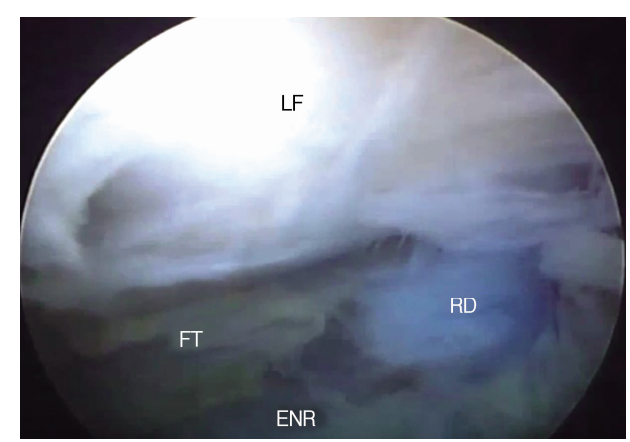

Fig. 2. Intraoperative endoscopic view (LF: splitted ligamentum flavum, RD: ruptured disc, FT: perineural fatty tissue, ENR: exiting nerve root).
USA). A blue-stained ruptured disc compressing an exiting nerve root was identified (Fig. 2). The ruptured disc was removed with endoscopic forceps (Fig. 3). After decompression of the lesion and bleeding control, the scope was removed. Decompression of the lesion was confirmed with post-operative MRI.

\section{RESULTS}

The average age of five patients was $55.8 \pm 10.04$ years. Three of them were male, two were female. The average VAS score was reduced from $7.8 \pm 0.84$ pre-operatively to $1.4 \pm 0.55$ post-operatively (Table 1). All cases showed that the ruptured disc had been successfully removed on post-operative MRI. There were no complications such as dysthesia, hematoma, or infection. None of the cases needed any additional surgery.

\section{Illustrative case}

A 43-year-old man complained of a radiating pain to left leg, which had not responded to a conservative treatment. On preoperative MRI, L5-S1 foraminal disc herniation with superior migration compressed left L5 nerve root (Fig. 4A). A working channel was inserted towards the medial border of inferior articular process of the lesion (Fig. 4B). The ligamentum flavum was splitted, blue-stained ruptured disc was identified and removed (Fig. 4C). Post-operative MRI showed that the ruptured disc had been successfully removed and left L5 nerve root was well decompressed (Fig. 4D).

Table 1. Patients characteristics

\begin{tabular}{cccccc}
\hline \hline \multirow{2}{*}{ Patients } & \multirow{2}{*}{ Age } & \multirow{2}{*}{ Sex } & \multirow{2}{*}{ Migrated side } & \multicolumn{2}{c}{ VAS } \\
\cline { 5 - 6 } & & & Preop & Postop \\
\hline A & 43 & M & Left & 9 & 1 \\
B & 47 & F & Left & 8 & 2 \\
C & 61 & M & Right & 8 & 1 \\
D & 64 & F & Left & 7 & 1 \\
E & 64 & M & Left & 7 & 2 \\
\hline
\end{tabular}

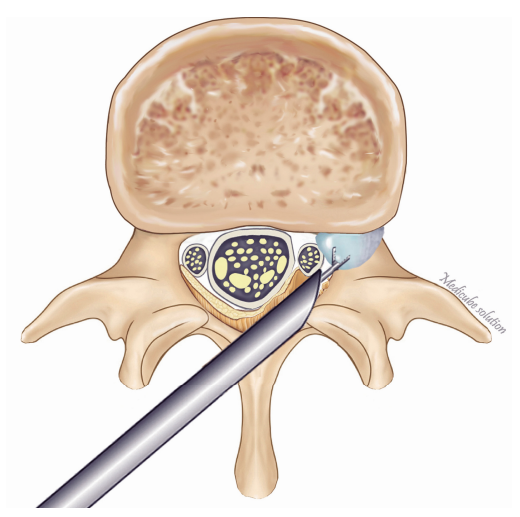

Fig. 3. Removal of ruptured disc with endoscopic forcep. 

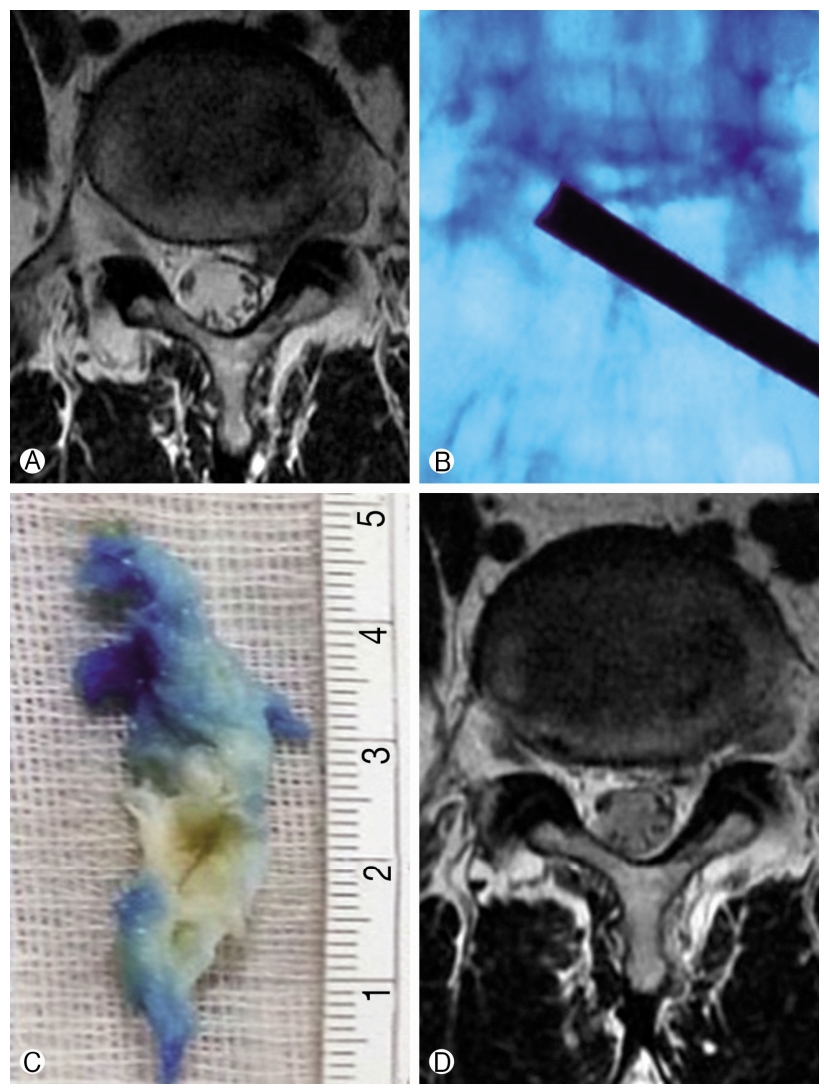

Fig. 4. Illustrative case. (A) Pre-operative MRI, (B) C-arm image, (C) Removed disc, (D) Post-operative MRI.

\section{DISCUSSION}

Since Kambin et al. first introduced lumbar disc decompression via a posterolateral approach ${ }^{7)}$, there have been remarkable advances in percutaneous endoscopic lumbar disc surgery. With the development of various instruments related to spinal endoscopic surgery, now nearly all symptomatic lumbar disc herniation can be operated via endoscope.

As L5-S1 level has considerable anatomical features, such as high iliac crest, facet joint hypertrophy, relatively narrow foramen, and wide interlaminar space. Therefore, several approaches to ruptured disc at the L5-S1 level have been considered, such as transforaminal, ipsilateral interlaminar, and transiliac appoaches ${ }^{3,6,12)}$. Choi et al. compared the transforaminal approach to the interlaminar approach in detail, depending on the location of lesion, but limited the lesion to intracanal disc herniation and did not include foraminal disc lesions ${ }^{6}$.

In particular, it is difficult to approach L5-S1 foraminal disc herniation with superior migration with rigid endoscope, and such approaches have not been reported yet. Using an excessive cranial to caudal skin entry point due to the high iliac crest increases the possibility of encountering an existing nerve root injury. It is also difficult to approach L5-S1 foraminal disc herniation with superior migration via the ipsilateral interlaminar appro- ach due to the inferior facet and laminar. Thus, microscopic lumbar discectomy via a paramedian transmuscular approach has been used for this type of lesion. However, microscopic approaches require partial facetectomy; if more than 40-50\% of facet is removed, instability may arise, inducing postoperative pain $^{2,8)}$.

The discs, facet joints, supraspinous ligaments, and paraspinal muscles of the lumbar spine are important structures that maintain a stability of segmental motion. Instability can be increased when these surrounding structures are damaged ${ }^{10,11)}$. PELD via a contralateral interlaminar approach can maximally preserve these structures.

If the interlaminar space is relatively narrow, it might render an approach to superior migration difficult, as the laminar would block the trajectory. In such a case, an approach trajectory can be acquired by undercutting the inferior margin of the lesionside facet using an endoscopic drill. Fortunately, in the present cases, we were able to approach the contralateral foramen without using an endoscopic drill. The outcomes were satisfying in all of cases, however the number of cases was small. Further study will be needed with more cases.

Since insertion of the working channel at a sharp angle may cause injury to the thecal sac, it is important to dock the working channel to the medial border of the inferior facet of lesions safely at an approximately 45-degree angle. In addition, splitting a ligamentum flavum without clarifying the boundaries of the bone and ligamentum flavum may cause exiting nerve root injury, thereby requiring attention.

\section{CONCLUSION}

This study achieved a good result through a contralateral interlaminar approach for L5-S1 foraminal disc herniation with superior migration. This can be a considerable option for the treatment of ruptured discs that had previously been difficult to approach with a rigid endoscope.

\section{REFERENCES}

1. Andrews DW, Lavyne MH: Retrospective analysis of microsurgical and standard lumbar discectomy. Spine (Phila Pa 1976) 15: 329-335, 1990

2. Bae JS, Kang KH, Park JH, Lim JH, Jang IT: Postoperative Clinical Outcome and Risk Factors for Poor Outcome of Foraminal and Extraforaminal Lumbar Disc Herniation. J Korean Neurosurg Soc 59:143-148, 2016

3. Choi G, Kim JS, Lokhande P, Lee SH: Percutaneous endoscopic lumbar discectomy by transiliac approach: a case report. Spine (Phila Pa 1976) 34:E443-446, 2009

4. Choi G, Lee SH, Bhanot A, Raiturker PP, Chae YS: Percutaneous endoscopic discectomy for extraforaminal lumbar disc herniations: extraforaminal targeted fragmentectomy technique using working channel endoscope. Spine (Phila Pa 1976) 32:E93-99, 2007

5. Choi G, Lee SH, Raiturker PP, Lee S, Chae YS: Percutaneous endoscopic interlaminar discectomy for intracanalicular disc her- 
niations at L5-S1 using a rigid working channel endoscope. Neurosurgery 58:ONS59-68; discussion ONS59-68, 2006

6. Choi KC, Kim JS, Ryu KS, Kang BU, Ahn Y, Lee SH: Percutaneous endoscopic lumbar discectomy for L5-S1 disc herniation: transforaminal versus interlaminar approach. Pain Physician 16: 547-556, 2013

7. Kambin P, Sampson S: Posterolateral percutaneous suction-excision of herniated lumbar intervertebral discs. Report of interim results. Clin Orthop Relat Res 207(19):37-43, 1986

8. Kotil K, Akcetin M, Bilge T: A minimally invasive transmuscular approach to far-lateral L5-S1 level disc herniations: a prospective study. J Spinal Disord Tech 20:132-138, 2007

9. Mayer HM, Brock M: Percutaneous endoscopic discectomy: surgical technique and preliminary results compared to microsurgical discectomy. J Neurosurg 78:216-225, 1993
10. Panjabi MM: The stabilizing system of the spine. Part I. Function, dysfunction, adaptation, and enhancement. J Spinal Disord 5:383-389; discussion 397, 1992

11. Panjabi MM: The stabilizing system of the spine. Part II. Neutral zone and instability hypothesis. J Spinal Disord 5:390-396; discussion 397, 1992

12. Ruetten S, Komp M, Godolias G: A New full-endoscopic technique for the interlaminar operation of lumbar disc herniations using 6-mm endoscopes: prospective 2-year results of 331 patients. Minim Invasive Neurosurg 49:80-87, 2006

13. Ruetten S, Komp M, Merk H, Godolias G: Full-endoscopic interlaminar and transforaminal lumbar discectomy versus conventional microsurgical technique: a prospective, randomized, controlled study. Spine (Phila Pa 1976) 33:931-939, 2008 